\title{
Using local fields to tailor hybrid quantum-dot/metal nanoparticle systems
}

\author{
Ryan D. Artuso* \\ Joint Quantum Institute and Department of Physics, University of Maryland, College Park, Maryland 20742-4111, USA
}

Garnett W. Bryant

Joint Quantum Institute and Atomic Physics Division, National Institute of Standards and Technology, Gaithersburg, Maryland 20899-8423, USA

Aitzol Garcia-Etxarri and Javier Aizpurua

Donostia International Physics Center and Centro de Fisica de Materiales CSIC-UPV/ EHU, Paseo Manuel de Lardizabal 4, Donostia-San Sebastian 20018, Spain

(Received 5 January 2011; revised manuscript received 11 March 2011; published 8 June 2011)

\begin{abstract}
We study hybrid-molecule structures consisting of a metal nanoparticle (MNP) nanorod coupled to a semiconductor quantum dot (SQD). MNP geometry can be used to tailor the local fields that determine the SQD-MNP coupling and to engineer the hybrid dynamical response. We identify regimes where dark modes and higher-order multipolar modes can influence hybrid response. Strong local-field coupling via dark modes changes the interference and self-interaction effects dramatically. External fields do not directly drive this MNP dark mode, so SQD-MNP coupling is dominated by the local induced self-coupling. By utilizing more complex structures that provide substantial local-field enhancement, we show the strong SQD-MNP coupling regimes can be made much more accessible. All of these aspects could enhance the capabilities of metal nanostructures to provide spatial and spectral control of the optical properties of single emitters.
\end{abstract}

DOI: 10.1103/PhysRevB.83.235406

PACS number(s): 78.67.Bf, 78.67.Lt, 42.50.-p

\section{INTRODUCTION}

Hybrid structures consisting of a semiconducting quantum dot (SQD) and a metal nanoparticle (MNP) have recently become a very active area of research. ${ }^{1-21}$ Such structures should allow for the directed nanoscale transmission of information and excitations. By coupling the plasmonic response of the MNP to the excitons of the SQD, these structures stimulate the study of systems at the interface between classical and quantum physics. Experimental investigations have shown efficient exciton-plasmon-photon conversion and an enhanced emission rate with the coupling of a CdSe quantum dot to a silver nanowire. ${ }^{2,3}$ When coupled to elongated MNPs, the photoluminescence intensity of SQDs is enhanced in a polarization-selective way, ${ }^{4}$ and when coupled with a nanooptical Yagi Uda antenna the SQD emission can be made unidirectional. ${ }^{5}$ Exciting results have been attained showing that quantum coherence can survive in plasmonic structures, such as the transportation of entangled photons by surface plasmons $^{6}$ and the energy-time entanglement of a pair of photons following a photon-plasmon-photon conversion. ${ }^{7}$

Previous theoretical investigations have mainly focused on spherical MNPs coupled with a SQD, in the weak-coupling regime $^{8}$ and in the strong-coupling regime. ${ }^{9-11}$ This system has been explored as a means to make ultrafast tunable nanoswitches. ${ }^{12}$ Similar systems have been studied with multiple metal nanoparticles ${ }^{13}$ and with dipole-dipole coupling between two fluorescent molecules mediated by a chain of silver nanoparticles. ${ }^{14}$ Plasmon-induced transparency has been studied in a system consisting of a three-level SQD interacting with a spherical MNP. ${ }^{15}$

Theoretical studies have also considered the coupling of SQDs to elongated MNPs, nanowires, and nanowaveguides, showing that the radiative rate of the exciton and the non- radiative energy-transfer rate are tunable by means of the aspect ratio for an $\mathrm{Au}$ nanorod coupled to a SQD. ${ }^{16}$ The coupling of two quantum emitters by means of a nanowire has been studied, showing Dicke subradiance and superradiance, leading to the possibility of a two-qubit quantum gate, ${ }^{17}$ and the entanglement of two qubits coupled by a wedge acting as a nanowaveguide has been explored. ${ }^{18}$ Similarly, a single emitter coupled to a plasmonic cavity ${ }^{19}$ and a comparative study of resonance energy transfer for a quantum emitter coupled to a nanowire, a nanowedge, or a channel ${ }^{20}$ has been carried out. Furthermore, the coupling of excitons to dark plasmonic states of elongated MNPs has been studied. ${ }^{21}$

The geometry of these hybrid systems determines the nature of the local fields, which in turn determines the couplings and the system behavior. Useful devices that will utilize these structures will require these more complicated geometries. To consider these more complex MNP structures, for example, nanorods and nanowires, chains of MNPs, and SQDs at various positions in the structure, a more complete model for the local fields needs to be employed than previously has been done for MNP spheres (i.e., typically using a nonretarded, dipole approximation for the MNP response). In this paper we will consider a MNP nanorod coupled to a SQD (see Fig. 1) to show how local fields can be tailored by MNP geometry both by enhancing the local fields and by providing additional MNP modes to control the MNP-SQD coupling that determines the hybrid response. We will utilize the boundary element method (BEM) so that we fully account for the local fields of such complex structures, including retardation and all multipolar orders.

There are two basic interactions involving the SQD. One is the coupling due to an applied plane-wave driving field (both directly with the driving field and from the response 


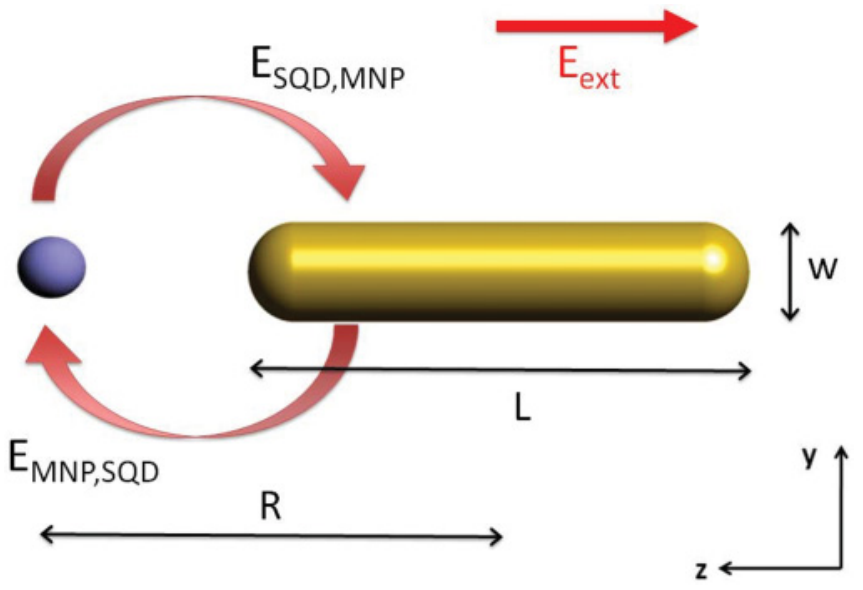

FIG. 1. (Color online) An applied field polarizes both the MNP and SQD, which in turn allows them to couple.

of the MNP to the applied plane wave). The second is the self-interaction of the SQD in which the MNP responds to the polarization field of the SQD and in turn produces a field felt back at the SQD. It was shown ${ }^{9,10}$ that the behavior of the system is determined by the ratio of these two couplings. This provides an avenue that can be exploited to engineer systems (by tuning both the coupling strength and geometry) to bias the response toward a particular regime of behavior, as we will discuss here. Previously, these two coupling strengths were varied by changing the radius of the MNP as well as the dipole moment of the SQD. However, in practical situations the SQD dipole moment could be difficult to control. It was shown ${ }^{9,10}$ that to reach the strongest-coupling regimes large and perhaps unreasonable values of the SQD dipole moment were needed. By utilizing structures with a more complex geometry that leads to substantial local-field enhancement, we show how even small values of the SQD dipole moment can be used to reach the strongest-coupling regimes. In addition, we identify MNP geometries where dark modes and higher-order multipolar modes can influence the response of the hybrid SQD-MNP system. External fields do not directly drive the MNP dark modes. Thus, at dark mode frequencies, SQD-MNP coupling is dominated by the local induced coupling, providing a situation in which the effects of the induced interaction can be enhanced relative to direct coupling to driving fields.

In Sec. II, we discuss the hybrid molecule in detail. The MNP is taken as a classical dielectric with a response calculated with the BEM. Upon finding the resultant fields acting on the SQD, we use a density-matrix approach to treat the SQD. Once the behavior of the SQD is found, we then use that information to calculate the local near fields in the immediate vicinity of our hybrid molecule. We also calculate the energy absorption of the MNP and provide details on how numerical calculations were carried out. In Sec. III, we show how the MNP local fields can be usefully engineered by the MNP geometry. We use full electrodynamical calculations to describe the MNP response. We first highlight the conditions where it is necessary to go beyond the nonretarded multipolardipolar limits, even for spherical particles. We then study the local-field response of a hybrid molecule made from a metal nanorod, stressing the local-field enhancement that can be achieved and the additional modes that can be exploited. In Sec. IV we discuss how coupling the SQD to a nanorod allows the system to be engineered toward a particular dynamical response. Here we exploit coupling to the dark states of the nanorod to investigate the enhancement of the self-interaction of the SQD. Finally, we present our conclusions in Sec. V.

\section{HYBRID MOLECULE}

We consider hybrid molecules consisting of a spherical SQD, radius $r$, interacting with a MNP structure which has cylindrical symmetry about the axis joining the SQD and the MNP structure. The MNP structure could be a nanorod or a nanosphere, as considered in Refs. 8-10, or a linear chain of those. In this paper we will consider the MNP to be a nanorod, constructed as a cylinder with semispherical ends, with a total length $L$ and width $w$ (see Fig. 1). The SQD and MNP are separated by a distance $R$. The entire system is subject to an applied electric field $E=E_{0} \cos (\omega t)$. We treat the SQD quantum mechanically in the density-matrix formalism with exciton energy $\hbar \omega_{0}$, dipole moment $\mu$, and dielectric constant $\epsilon_{S}$. In the dipole limit only the three bright excitons (one for each optical axis) participate in the interaction. By choosing the direction of the applied field to be either perpendicular or parallel to the axis of our system, we, in turn, only excite one of the three excitons. Dark excitons do contribute to the exciton lifetime, however.

The optical response of the MNP is calculated by means of the boundary element method in a full electromagnetic calculation, including retardation. Retardation must be included because we consider structures with lengths that can be comparable to the wavelength of incident light. In the BEM, Maxwell's equations for inhomogeneous media with sharp boundaries are solved in terms of charges and currents distributed on the surfaces and interfaces. Boundary conditions are imposed via surface integrals along the boundaries between different media. Each region is characterized by a local dielectric function. The external fields interact self-consistently with the induced boundary charges and currents, which are determined by discretizing the surface integrals and solving the appropriate matrix equations. In this approach, the scattered field due to an incident external field is calculated directly. We calculate, in this way, both the near and far fields for a given MNP from both a plane-wave source as well as from a dipole source (the SQD). In handling the dipole source, we take an adiabatic approach and do not include the time dependence of the dipole moment in Maxwell's equations.

The Hamiltonian for the two-level SQD, $\mathcal{H}_{\mathrm{SQD}}$, is

$$
\mathcal{H}_{\mathrm{SQD}}=\hbar \omega_{0} \hat{a}^{\dagger} \hat{a}-\mu E_{\mathrm{SQD}}\left(\hat{a}+\hat{a}^{\dagger}\right)
$$

where $\hat{a}$ and $\hat{a}^{\dagger}$ are the atomic, two-level operators representing exciton creation and annihilation (corresponding to the $z$ direction with the applied field parallel to the molecule). $E_{\mathrm{SQD}}$ is the total electric field felt by the SQD and consists of the applied external field $E$ and the induced internal field, produced by the polarization of the MNP. The MNP is polarized by the applied plane wave, and we denote the response field as $E_{\text {plane wave. }}$ The MNP is also polarized by the dipole field produced by the SQD, and this response field 
we denote $E_{\text {dipole }}$. Taking into account screening, we can then write $E_{\mathrm{SQD}}$ as

$$
E_{\mathrm{SQD}}=\frac{1}{\epsilon_{\mathrm{effS}}}\left(E+E_{\text {plane wave }}+E_{\text {dipole }}\right),
$$

where $\epsilon_{\mathrm{effS}}=\frac{2 \epsilon_{B}+\epsilon_{S}}{3 \epsilon_{B}} . \epsilon_{B}$ is a background dielectric constant which would correspond to the medium in which the system is embedded. Writing the applied field as $E=E_{0} \cos (\omega t)=$ $\frac{E_{0}}{2} e^{-i \omega t}+\frac{E_{0}}{2} e^{i \omega t}$ and the response from the MNP due to a unit plane wave with positive frequency, at the location of the SQD, as $E_{p}$ (which will be calculated using the BEM and in general depends on $R, L$, and $w$ ), then

$$
E_{\text {plane wave }}=\frac{E_{0}}{2} E_{p} e^{-i \omega t}+\frac{E_{0}}{2} E_{p}^{*} e^{i \omega t} .
$$

We make use of the density matrix $\rho$ to calculate the polarization of the SQD. We label the ground state of our SQD (no exciton) as level 1 and the excited state (one exciton) we label as level 2 . We then have for the polarization, $P_{\mathrm{SQD}}=$ $\mu\left(\rho_{12}+\rho_{21}\right)$ (see Ref. 22). Factoring out the high-frequency time dependence of the off-diagonal terms of the density matrix, we define

$$
\rho_{12}=\widetilde{\rho}_{12} e^{i \omega t} \quad \rho_{21}=\widetilde{\rho}_{21} e^{-i \omega t} .
$$

We write the response of the MNP due to a dipole source with positive frequency at the location of the SQD as $E_{d}$ (which again will be calculated using the BEM and in general depends on $R, L$, and $w$ ). Taking into account the screening at the dipole, we then have

$$
E_{\text {dipole }}=\frac{\mu}{\epsilon_{\mathrm{effS}}} \widetilde{\rho}_{21} E_{d} e^{-i \omega t}+\frac{\mu}{\epsilon_{\mathrm{effS}}} \widetilde{\rho}_{12} E_{d}^{*} e^{i \omega t} .
$$

Putting these expressions for $E_{\text {plane wave }}$ and $E_{\text {dipole }}$ into Eq. (2), as well as writing out the applied field explicitly, we have

$$
E_{\mathrm{SQD}}=\frac{1}{\epsilon_{\mathrm{effS}}}\left(\frac{E_{0}}{2}\left(1+E_{p}\right)+\frac{\mu}{\epsilon_{\mathrm{effS}}} \widetilde{\rho}_{21} E_{d}\right) e^{-i \omega t}+\text { H.c.. }
$$

In order to write this in a more familiar form ${ }^{8-10}$ we then make the following definitions:

$$
G=\frac{\mu^{2}}{\hbar \epsilon_{\mathrm{effS}}^{2}} E_{d}, \quad \Omega=\frac{\mu E_{0}}{2 \hbar \epsilon_{\mathrm{effS}}}\left(1+E_{p}\right) .
$$

$G$ arises when the applied field polarizes the SQD, which in turn polarizes the MNP and then produces a field to interact with the SQD. Thus, this can be thought of as the self-interaction of the SQD because this coupling to the SQD depends on the polarization of the SQD. The first term in $\Omega$ is just the direct coupling to the applied field and the second term is the field from the MNP that is induced by the applied field. We can now write the field acting on the SQD as

$$
E_{\mathrm{SQD}}=\frac{\hbar}{\mu}\left\{\left(\Omega+G \widetilde{\rho}_{21}\right) e^{-i \omega t}+\left(\Omega^{*}+G^{*} \widetilde{\rho}_{12}\right) e^{i \omega t}\right\} .
$$

We solve the master equation

$$
\dot{\rho}=\frac{i}{\hbar}\left[\rho, \mathcal{H}_{\mathrm{SQD}}\right]-\Gamma(\rho),
$$

where $\Gamma(\rho)$ is the relaxation matrix with entries $\Gamma_{11}=$ $\frac{\rho_{11}-1}{\tau_{0}}, \Gamma_{12}=\Gamma_{21}^{*}=\frac{\rho_{12}}{T_{20}}$, and $\Gamma_{22}=\frac{\rho_{22}}{\tau_{0}}$. The relaxation time $\tau_{0}$ contains a contribution from nonradiative decay to dark states. Separating real and imaginary parts, we now write the density-matrix elements as

$$
\tilde{\rho}_{12}=A+i B, \quad \widetilde{\rho}_{21}=A-i B, \quad \Delta=\rho_{11}-\rho_{22},
$$

where $\Delta$ is the population difference between the excited and ground states. To solve (6), we make the rotating wave approximation. When changing the Hamiltonian to the interaction picture we keep terms that oscillate as $e^{i\left(\omega-\omega_{0}\right) t}$ and neglect terms that oscillate as $e^{i\left(\omega+\omega_{0}\right) t}$. Making use of our definitions and the rotating wave approximation, we come to the set of coupled differential equations,

$$
\begin{gathered}
\dot{A}=-\frac{A}{T_{20}}+\left(\omega-\omega_{0}\right) B-\left(\Omega_{I}+G_{I} A-G_{R} B\right) \Delta, \\
\dot{B}=-\frac{B}{T_{20}}-\left(\omega-\omega_{0}\right) A-\left(\Omega_{R}+G_{R} A+G_{I} B\right) \Delta, \\
\dot{\Delta}=\frac{1-\Delta}{\tau_{0}}+4 \Omega_{I} A+4 \Omega_{R} B+4 G_{I}\left(A^{2}+B^{2}\right),
\end{gathered}
$$

where $G_{R}, G_{I}, \Omega_{R}$, and $\Omega_{I}$ are the real and imaginary parts of $G$ and $\Omega$, respectively.

In the steady-state limit we set the left-hand side of (7) to zero. Due to the nonlinear nature of these equations, more than one steady-state solution can exist for certain values of the parameters. In these regions we must solve the full set of dynamical equations (7), allowing them to evolve from the initial conditions for times on the order of $10 \mathrm{~ns}$ to reach the steady state. This allows us to identify the dependence of the steady state on the starting conditions. Except where noted, evolution for $10 \mathrm{~ns}$ was sufficient to reach steady state in the cases we considered.

\section{A. System energy}

The rate at which energy is absorbed by our system consists of two parts, $Q_{\mathrm{SQD}}$ and $Q_{\mathrm{MNP}}$. The SQD absorbs energy by the creation of an exciton followed by a nonradiative decay. The rate is just $Q_{\mathrm{SQD}}=\hbar \omega_{0} \rho_{22} / \tau_{0}$. To calculate the energy absorbed by the MNP, we take the time average of the volume integral, $\int \mathbf{j} \cdot \mathbf{E} d v$, where $\mathbf{j}$ is the local current density. To calculate the fields inside the MNP we again employ the BEM. We denote the field inside the MNP due to the dipole field of the SQD as $E_{\text {dipole }}^{\text {(inside) }}$ This field includes the field due to the polarization induced in the MNP as well as the dipole field of the SQD. Similarly, we denote the field inside the MNP due to the applied plane wave as $E_{\text {plane wave }}^{\text {(inside) }}$, and this field includes the field due to the polarization induced in the MNP as well as the applied field. These fields with positive frequency can be determined once $E_{d}^{i}$ (the field inside the MNP due to a unit dipole with positive frequency at the SQD) and $E_{p}^{i}$ (the field inside the MNP due to a unit incident plane wave with positive frequency) are known. We write

$$
\begin{gathered}
E_{\text {dipole }}^{\text {(inside) }}=\frac{\mu}{\epsilon_{\text {effS }}} \widetilde{\rho}_{21} E_{d}^{i} e^{-i \omega t}+\frac{\mu}{\epsilon_{\text {effs }}} \widetilde{\rho}_{12}\left(E_{d}^{i}\right)^{*} e^{i \omega t}, \\
E_{\text {plane wave }}^{\text {(inside) }}=\frac{E_{0}}{2} E_{p}^{i} e^{-i \omega t}+\frac{E_{0}}{2}\left(E_{p}^{i}\right)^{*} e^{i \omega t},
\end{gathered}
$$

in a notation similar to what we have previously employed. We note that $E_{p}^{i}$ and $E_{d}^{i}$ both depend on $R, L, w$, and the 
position in space at which the field is calculated. Thus the field inside the MNP is just the sum of these two fields. In order to calculate the current inside the MNP, we take the curl of the magnetic field to be zero and use $j=-\epsilon_{m}(\omega) \frac{\partial E}{\partial t}$. Because we are assuming that we have factored out the fast varying part of the density matrix in (3), we take the time derivatives of $\widetilde{\rho}_{12}$ and $\tilde{\rho}_{21}$ to be zero, and we then have for the local current $j$,

$$
\begin{aligned}
j= & i \omega\left\{\epsilon_{m}(\omega)\left(\frac{E_{0}}{2} E_{p}^{i}+\frac{\mu}{\epsilon_{\mathrm{effS}}} \widetilde{\rho}_{21} E_{d}^{i}\right) e^{-i \omega t}\right. \\
& \left.-\epsilon_{m}(\omega)^{*}\left(\frac{E_{0}}{2}\left(E_{p}^{i}\right)^{*}+\frac{\mu}{\epsilon_{\mathrm{effS}}} \widetilde{\rho}_{12}\left(E_{d}^{i}\right)^{*}\right) e^{i \omega t}\right\} .
\end{aligned}
$$

We can now calculate $\mathbf{j} \cdot \mathbf{E}$,

$$
\begin{aligned}
\mathbf{j} \cdot \mathbf{E}= & i \omega\left\{\epsilon_{m}(\omega)\left(\frac{E_{0}}{2} E_{p}^{i}+\frac{\mu}{\epsilon_{\mathrm{effS}}} \widetilde{\rho}_{21} E_{d}^{i}\right)^{2} e^{-2 i \omega t}\right. \\
& +\epsilon_{m}(\omega)^{*}\left(\frac{E_{0}}{2}\left(E_{p}^{i}\right)^{*}+\frac{\mu}{\epsilon_{\mathrm{effS}}} \widetilde{\rho}_{12}\left(E_{d}^{i}\right)^{*}\right)^{2} e^{2 i \omega t} \\
& \left.+2 i \operatorname{Im}\left[\epsilon_{m}(\omega)\right]\left|\frac{E_{0}}{2}\left(E_{p}^{i}\right)+\frac{\mu}{\epsilon_{\mathrm{effS}}} \widetilde{\rho}_{12}\left(E_{d}^{i}\right)\right|^{2}\right\} .
\end{aligned}
$$

Taking the time average of this result over the period of fast oscillation and integrating over the volume of the MNP yields $Q_{\text {MNP }}$

$$
Q_{\mathrm{MNP}}=2 \omega \operatorname{Im}\left[\epsilon_{m}(\omega)^{*}\right] \int\left|\frac{E_{0}}{2}\left(E_{p}^{i}\right)+\frac{\mu}{\epsilon_{\mathrm{effS}}} \widetilde{\rho}_{21}\left(E_{d}^{i}\right)\right|^{2} d v .
$$

We calculate this integral numerically using the BEM to find $\left(E_{p}^{i}\right)$ and $\left(E_{d}^{i}\right)$ and Eq. (7) to find $\widetilde{\rho}_{21}$ in the steady-state limit.

\section{B. Numerical calculations in the large field limit}

In this paper we take our MNP to have cylindrical symmetry about the $z$ axis and we take the dielectric constant of the background to be $\epsilon_{B}=\epsilon_{0}$. We model the MNP as a cylinder with hemispherical ends, with total length $L$ and total width $w$ (see Fig. 1). Thus, in the case of $L=w$, we have a sphere of radius $w / 2$. The SQD is placed on the positive $z$ axis a distance $R$ from the center of the MNP. We consider the large field limit as defined in Ref. 8 (intensity of $10^{3} \mathrm{~W} / \mathrm{cm}^{2}$ ) and we take the polarization of the applied electric field parallel to the axis of our SQD-MNP molecule (the $\hat{z}$ direction) with the propagation along the $\hat{x}$ direction. We take $\epsilon_{m}(\omega)$ as the bulk dielectric constant of gold as found experimentally. ${ }^{23}$

For the SQD, we take the relaxation times to be $\tau_{0}=0.87 \mathrm{~ns}$ and $T_{20}=0.3 \mathrm{~ns}$, and we take $\epsilon_{S}=6 \epsilon_{0}$. For the the exciton resonant frequency we take it to be in the range of $1-4 \mathrm{eV}$ in order to exploit potentially matching this with the plasmon resonances of the nanorods we will study. However, the size, shape, and material of the SQD strongly determine both the exciton energy level and its dipole moment. In this paper, we consider the simplest model and ignore this size dependence. While this is clearly an oversimplification, it allows us to identify the range of optical signatures which are possible in this limit. Recent measurements of SQD dipole moments have yielded values of $\approx 1 e \mathrm{~nm}$ for self-assemble $\mathrm{QDs}^{24}$ and several times that for interface fluctuation QDs. ${ }^{25}$ For the dipole moments of the SQDs, we let them vary between
0.25 and $1.0 e \mathrm{~nm}$, corresponding to a SQD size of 2-10 $\mathrm{nm}$. For the purposes of this investigation, this range is a reasonable coverage of the observed values that allows us to test the full spectrum of behavior.

\section{ENGINEERED LOCAL FIELDS AND COUPLINGS}

We use full electrodynamical calculations to describe systems with complicated geometry. We do this in part because such approaches are straightforward to implement, as compared to multipolar expansion, for example, for these structures. This allows us to study any shape of MNP we choose. Additionally, one can study the effects of placing the SQD off the symmetry axis. Moreover, the multipole expansion only has a simple form in the cases that the applied field is perpendicular or parallel to the molecular axis. Moreover, the simpler nonretarded multipolar or dipolar approaches are not always adequate, even for spherical particles, where they are usually applied. In Sec. III A, we discuss the limits of the nonretarded approaches. In Sec. III B, we show how local fields can be tuned by MNP geometry.

\section{A. Comparison between a full electrodynamical calculation and a nonretarded multipole expansion for spherical MNPs}

Previously, calculations have been done on a system consisting of a SQD interacting with a spherical MNP in the dipole limit ${ }^{8-10}$ as well as in the multipole limit, ${ }^{11}$ both of which are nonretarded approaches. In the dipole limit, the ratio of MNP radius $a$ to MNP-SQD separation $R$ should be small [as the multipole expansion is a power series in $\left(\frac{a}{R}\right)^{2}$ ]. For $a=7 \mathrm{~nm}, R=13 \mathrm{~nm}$, the first-order correction is nearly $30 \%$.

It was shown ${ }^{11}$ that for $a=15 \mathrm{~nm}, R=20 \mathrm{~nm}$, the sum of the first ten terms of the multipole expansion of the coupling strength between the MNP and SQD is almost seven times greater than that given by only the first term in the series (the dipole limit). The most interesting hybrid molecules are those with strong coupling. One manner of increasing the coupling strength between the MNP and SQD is to place the SQD very close to the MNP, and thus the correction due to higher-order multipole terms will be important in the systems we most wish to study.

In order to compare the results obtained using the BEM with those given by the multipole expansion, we compare the values of $G$ given by the two methods, because there is no multipolar correction to the response due to the applied plane wave. In the multipole limit, $G$ is given by the expression ${ }^{11}$

$$
G=\sum_{n=1}^{\infty} \frac{s_{n} \gamma_{n} a^{2 n+1} \mu^{2}}{4 \pi \epsilon_{B} \hbar \epsilon_{\mathrm{effS}}^{2} R^{2 n+4}},
$$

where

$$
\gamma_{n}=\frac{\epsilon_{m}(\omega)-\epsilon_{B}}{\epsilon_{m}(\omega)+\frac{n+1}{n} \epsilon_{B}},
$$

and $s_{n}=(n+1)^{2}$ or $P_{n}^{\prime}(1)$ for polarization parallel or perpendicular to the $z$ axis and $P_{n}^{\prime}$ is the first derivative of the Legendre function. When we take only the term with $n=1$, we recover the dipole approximation. In order to calculate the fields in the multipole expansion for systems where the total separation is 


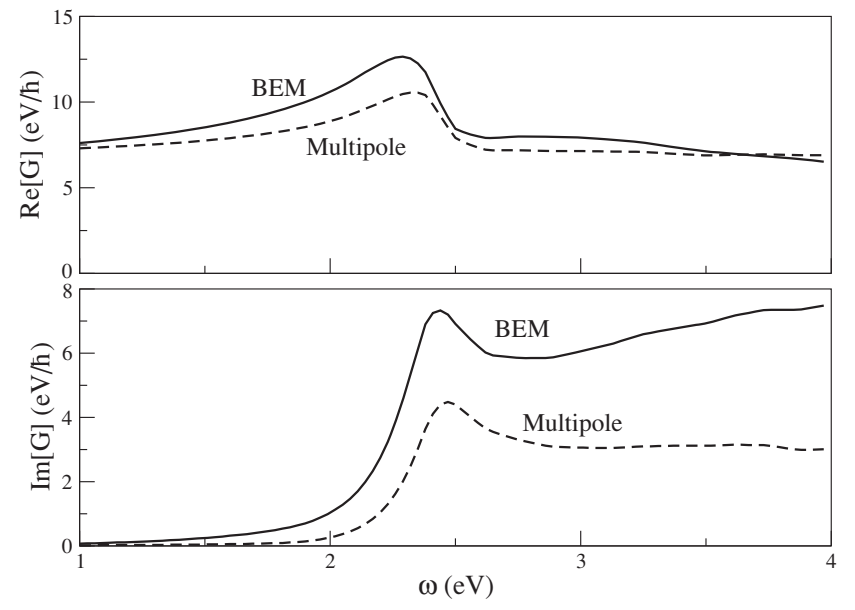

FIG. 2. The real and imaginary parts of $G$ with $R=60 \mathrm{~nm}$ and $a=40 \mathrm{~nm}$, shown for both a full electrodynamical calculation using the boundary element method (labeled as BEM) and a nonretarded multipole expansion (labeled Multipole). In this case we see an enhancement in the imaginary part of $G$, and a slight enhancement and redshift of the peak in the real part of $G$.

very nearly equal to the radius of the $\operatorname{MNP}(R \approx a)$, as many as 500 terms were needed for convergence of (8).

For a spherical MNP with $R=60 \mathrm{~nm}$ and $a=40 \mathrm{~nm}$, we see an enhancement in the imaginary part of $G$ of nearly a factor of 2 . We also see a slight enhancement of $\approx 25 \%$ and a redshift on the order of $0.1 \mathrm{eV}$ in the peak of the real part of $G$ (see Fig. 2). When this calculation is carried out over a large subset of MNP sizes and separation, we see that this enhancement increases as the MNP radius is increased for fixed separation up to a MNP radius of $\sim 50 \mathrm{~nm}$ (except in the most extreme cases of very small separation) or if the separation is increased for fixed MNP radius (see Fig. 3). However, as the separation between the SQD and the surface of MNP becomes small $(R \approx a)$, the calculations from the boundary element method and the multipole expansion yield the same results.

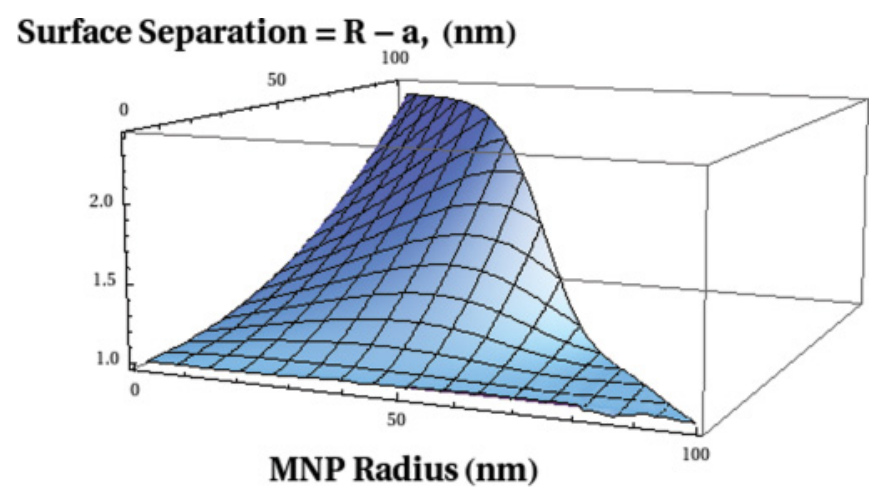

FIG. 3. (Color online) Ratio of the magnitude of $G$ as calculated with a full electrodynamical calculation using the BEM to that of the multipole expansion at a frequency of $2.5 \mathrm{eV}$, which is near the plasmon resonance for an $\mathrm{Au}$ sphere. The value given by the BEM increases in relation to that of the multipole as the MNP radius is increased for fixed separation up to a MNP radius of $\sim 50 \mathrm{~nm}$ (except in the most extreme cases of very small separation) or if the separation is increased for fixed MNP radius.
We do, however, note that in these extreme cases, as many as 500 terms of the expansion were needed for the sum to converge.

To understand these results, it is beneficial to think in terms of the image charges induced in the MNP by the SQD rather than the self-interaction. The image charges induced in the MNP should arise from the polarization of the dipole mode of the MNP, with image charges at both ends of the MNP, one nearest to the SQD and one at the furthest point. In the retarded limit, these two image charges can be out of phase with each other owing to their spatial separation. For very small surfaceto-surface separation, the SQD is very near to the closer image charge, with much stronger coupling to that image charge, and much weaker coupling to the charge at the other end. In this case, any phase difference is unimportant and the retarded and nonretarded limits are the same, as seen on the near edge of Fig. 3. However, as the SQD and MNP are separated, the effect of the image charge on the backside of the MNP also becomes significant, screening the effect of the image charge nearest to the SQD. In the retarded limit, when there is a phase difference between these two image charges, the screening is less effective and thus a full electrodynamical calculation yields a larger value of the field than the nonretarded limit.

\section{B. From spheres to rods}

When we replace the spherical MNP with a nanorod, the effect on coupling can be quite dramatic (see Fig. 4). Holding the width of the nanorod fixed at $14 \mathrm{~nm}$, as we move from a rod of length $14 \mathrm{~nm}$ (which is a 7-nm radius sphere) to a rod of length $150 \mathrm{~nm}$, we see an enhancement in the value of $\Omega_{R}$ of approximately an order of magnitude, and of $\Omega_{I}$ of nearly 25 times at the peak value (see Fig. 4). Whereas the peak values of $G$ remain relatively unchanged, we do see higher-order modes appear in the spectrum. At $L=150 \mathrm{~nm}$ a total of four peaks have appeared in $G$, whereas there are only two in $\Omega$. We also note a redshift in the principle plasmon peak for both $G$ and $\Omega$.

As was shown previously, ${ }^{10}$ the ratios of the various components of $G$ and $\Omega$ are key in determining the behavior of the SQD-MNP molecule. The more complicated spectrum provided by a rod yields a much broader range of system behavior. The enhancement provided by a rod also allows for the regimes of strongest coupling to be more easily accessed experimentally.

\section{Coupling to dark states versus bright states}

As we increase the length of the nanorod, higher-order modes appear in the response. Even order dipole-forbidden modes, however, do not appear in the response to a plane-wave source $(\Omega)$, due to symmetry considerations, but they do appear in the response to a dipole source $(G)$. Such states are called dark states. Thus for an $L=150 \mathrm{~nm}$ nanorod, there are a total of four peaks that have appeared in $G$, whereas there are only two in $\Omega$. The two additional peaks in $G$ are thus dark.

For a $70-\mathrm{nm}$ rod, we see that both $G$ and $\Omega$ are peaked at $\sim 1.6 \mathrm{eV}$. However, we also see a strong response in $G$ between 2 and $2.5 \mathrm{eV}$, whereas there is no appreciable response in $\Omega$ over that range of frequencies (see Fig. 5). Thus, at this frequency, the SQD will only couple directly to the applied 

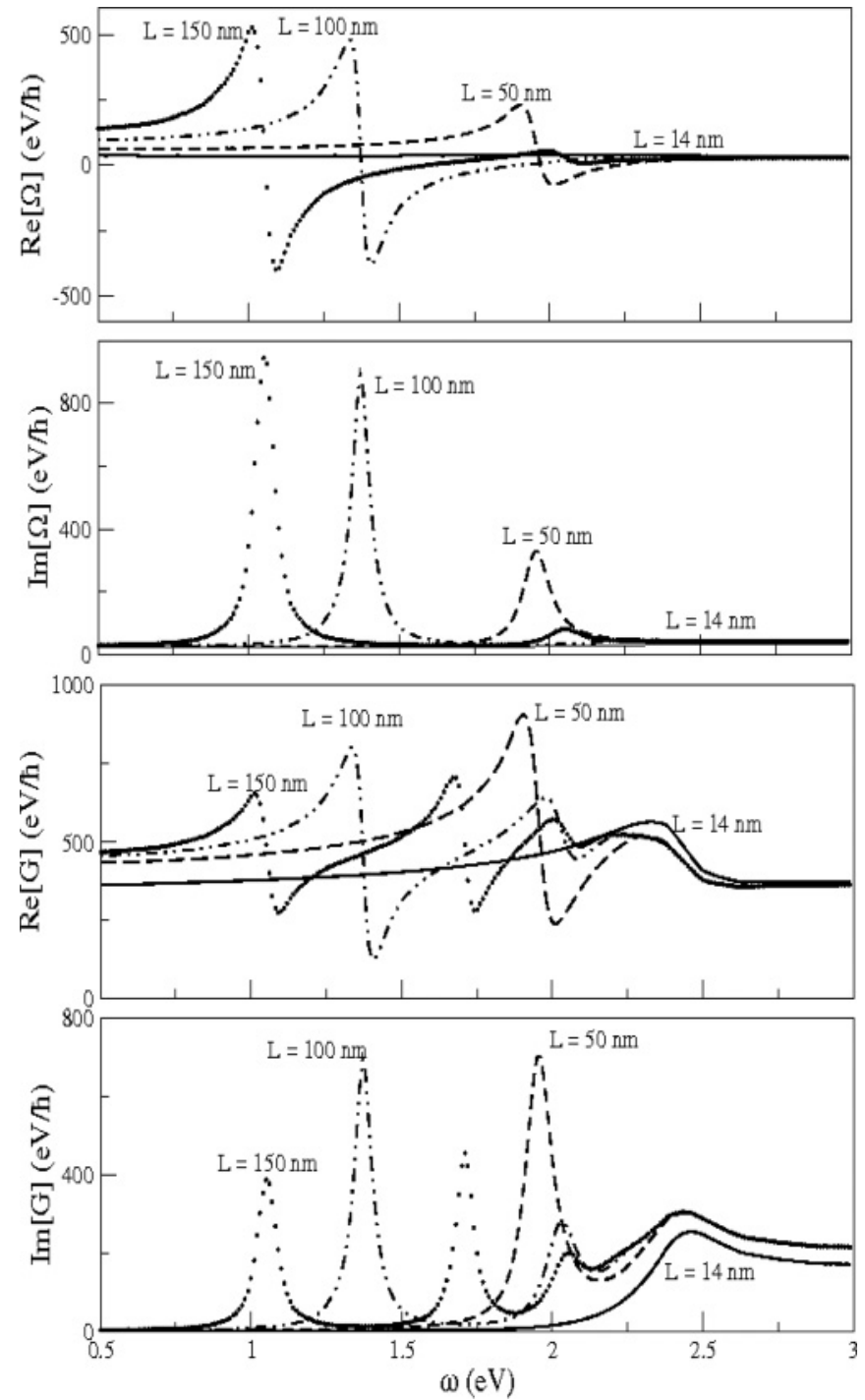

FIG. 4. The real and imaginary components of $G$ and $\Omega$ for various values of the rod length. The width of the nanorod was fixed at $14 \mathrm{~nm}$, thus the $L=14 \mathrm{~nm}$ setup is that of a $7-\mathrm{nm}$ radius sphere. $\Omega$ shows a redshift as well as an enhancement in the response as the length is increased. $G$ shows a redshift as well as the appearance of higher-order modes. At $L=150 \mathrm{~nm}$ a total of four peaks have appeared in $G$, whereas there are only two in $\Omega$.

field. However, the MNP is not polarized by the applied field, so the only coupling between the SQD and the MNP is via the self-interaction $(G)$. Coupling to these dark states will then allow us to study more closely the self-interaction of the SQD (which is controlled by $G$ ).

To better illustrate what occurs here, we next consider the near field response (time average of the squared magnitude of the electric field) of the nanorod to a plane wave and dipole source, respectively. We place the dipole source $5 \mathrm{~nm}$ above the tip of the rod with a dipole moment of $1 e \mathrm{~nm}$. The coupling to a plane-wave source in this situation can be a full order of magnitude smaller for a dark state as compared to that of a bright state (see Fig. 6). Whereas the bright state has regions of large electric field in the vicinity of each end of the rod (over 20 times that of the applied field), the dark state only has

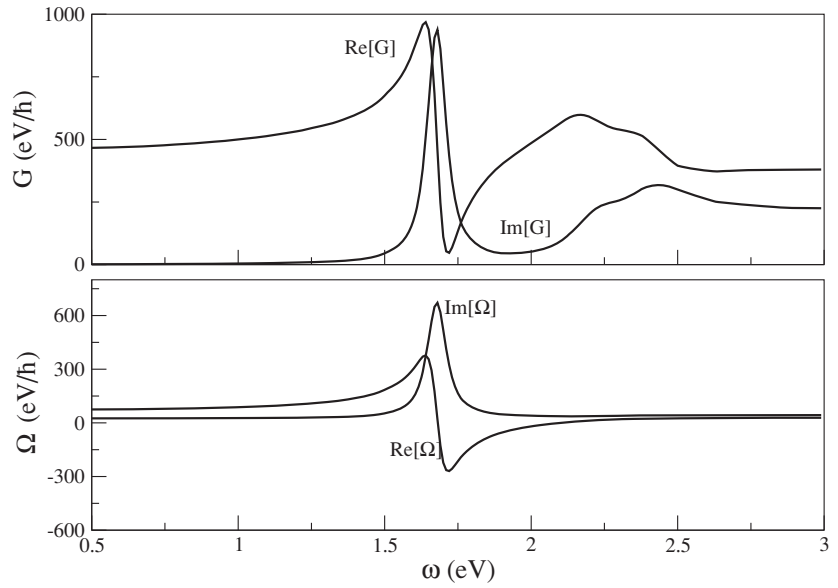

FIG. 5. Response of a 70-nm nanorod. Both $G$ and $\Omega$ are peaked near $1.6 \mathrm{eV}$. However, we also see a strong response in $G$ between 2 and $2.5 \mathrm{eV}$, whereas there is no appreciable response in $\Omega$ over that range of frequencies. The peak near $2.2 \mathrm{eV}$ in the real part of $G$ is evidently a "dark" mode.

a slight increase in field strength near the rod (about twice that of the applied field). However, the response to the dipole only shows a drop of approximately a factor of 2 , when comparing the response of the bright state to that of the dark state.

\section{ENGINEERED SYSTEM RESPONSE}

Once the values of $G$ and $\Omega$ are obtained, the differential equations for the SQD evolution [Eq. (7)] can be solved either dynamically, or in the steady-state limit. Once the density matrix is obtained, those results can then be used to calculate the SQD polarization, and from that the total electric field at any location in space, for any particular value of the applied plane-wave frequency. Furthermore, these local fields can be calculated at any instance of the system evolution.

\section{A. Dynamics of a 70-nm nanorod}

Previously 9,10 we have shown that by varying the SQD dipole moment and the radius of a spherical MNP, a large variety of system behavior is achievable. However, to reach the regimes of strongest coupling, relatively large values of the SQD dipole moment were needed, $\mu>3 e \mathrm{~nm} .{ }^{10}$ Such large values of the SQD dipole moment might not be experimentally possible due to the limit imposed by the SQD size and the relationship of SQD size to the exciton resonance. We will show for a nanorod, rather than a spherical MNP, that the large local response of the rod makes the strong-coupling regime accessible for smaller SQD dipole moments that are much more experimentally viable.

Here we illustrate a paradigm for designing systems biased toward specific behavior with the example of a 70-nm length nanorod with a total width of $14 \mathrm{~nm}$. We fix the SQD dipole moment at $\mu=0.5 e \mathrm{~nm}$. We have shown previously ${ }^{10}$ that suppression in the response of the SQD is controlled by the ratio of $G / \Omega$ and bistability in the system is turned on by the ratio $G_{I} / \Omega$. We thus use the values of these two ratios to predict system behavior at a given frequency. As shown in Fig. 7, for a 70-nm nanorod, these two ratios can take on a 

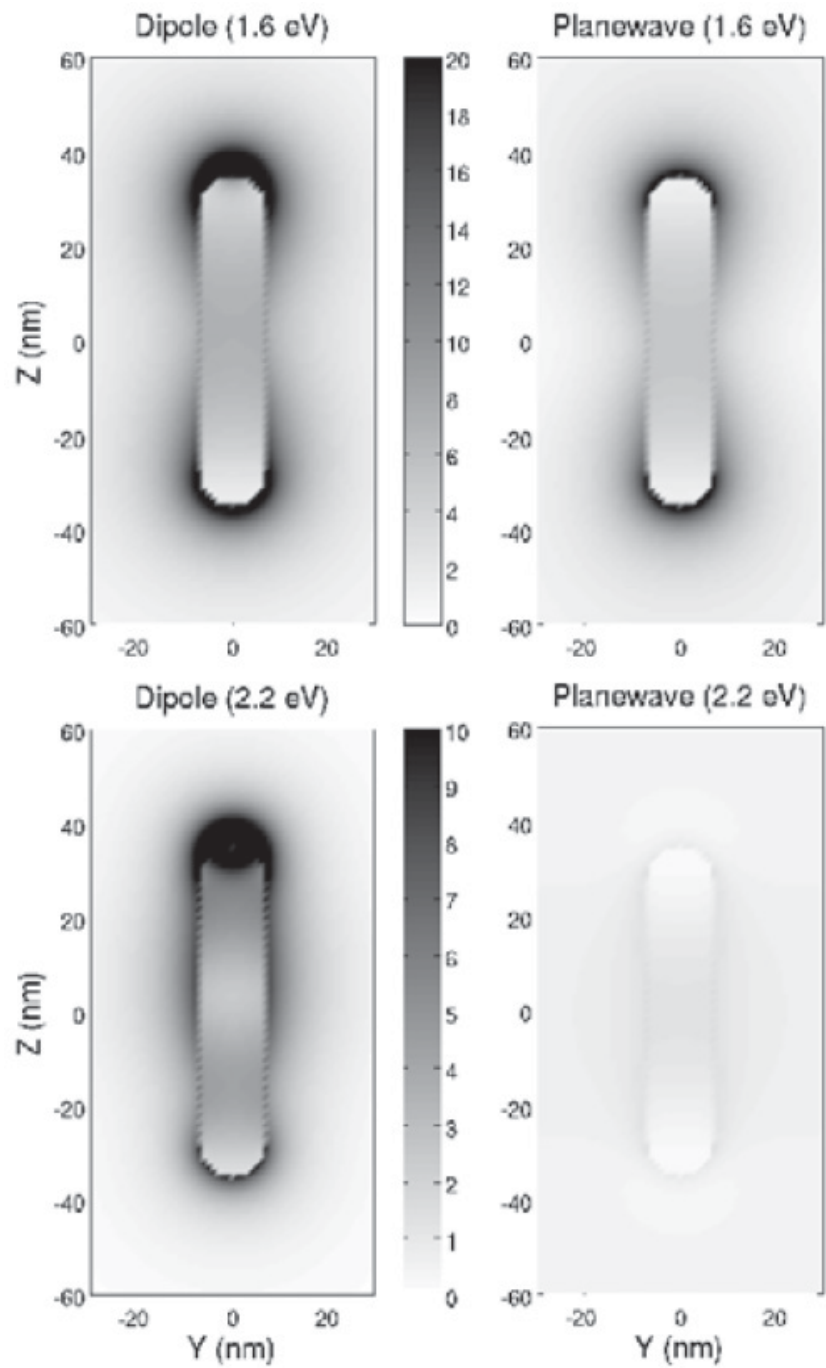

FIG. 6. Time average of the squared magnitude of the electric field of a 70-nm nanorod with a plane wave and dipole source. The dipole source was placed $5 \mathrm{~nm}$ above the tip of the rod with a dipole moment of $1 e \mathrm{~nm}$. (top) For the bright mode at $1.6 \mathrm{eV}$, there are hot spots in excess of 20 times the applied electric field for both the dipole and plane wave. (bottom) The dark mode at $2.2 \mathrm{eV}$ has a response to the dipole source (in excess of ten times that of reference field), however, the rod shows nearly no response to the plane-wave source.

larger range of values compared to those of a spherical MNP. In particular, the minimum of the ratio of the self-interaction to the coupling to the applied field, $G / \Omega$, is nearly a order of magnitude smaller ( 9 to 1 ) for the nanorod, while its maximum is $\sim 40 \%$ larger.

\section{Primary plasmonic peak (bright state)}

As shown in Fig. 6, the bright state exhibits a large response in the MNP due to the dipole of the SQD as well as to the plane wave. When the response of the nanorod is compared to that of a sphere, $\Omega$ is much larger than for a sphere (see Fig. 4). This enhancement of the local fields at the tips of the nanorod is simply due to a lightning rod effect. On the other hand, the differences in $G$ between a nanorod and sphere are

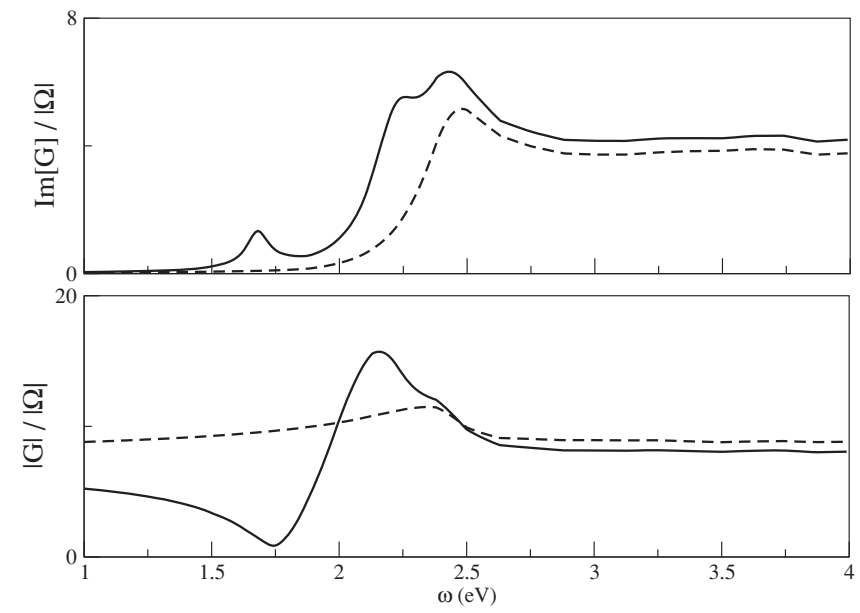

FIG. 7. The ratio of $|G| /|\Omega|$ and $G_{I} /|\Omega|$ shown for a 70-nm-long, 14-nm-wide nanorod (solid line) and a 7-nm radius spherical MNP (dashed line), with $\mu=1.0 e \mathrm{~nm}$. These two ratios play a large role in determining the system behavior.

small as compared to those in $\Omega$. Thus, for the bright state, we expect the SQD to couple more to the plane wave than the self-interaction, and, due to the local-field enhancement, to exhibit characteristics of much stronger applied field (such as increased width of the response).

When we take the exciton resonance to coincide with the bright plasmon peak $(\approx 1.6 \mathrm{eV})$, we see a very broad response in the SQD (see Fig. 8), as well as a broad Fano-like line shape in the absorption of the MNP, despite a rather modest value of the SQD dipole moment ( $\mu=0.5 e \mathrm{~nm})$. When we compare this to a system consisting of a spherical MNP and a similarly sized SQD, the width of the population difference $\Delta$ is a full order of magnitude larger for a nanorod of this length (1 meV as compared to $0.1 \mathrm{meV}$ ). This is due to the much stronger local near fields in the vicinity of the MNP incident on the SQD. This enhancement is due the increased response of the nanorod to a plane wave in comparison to that of a sphere.

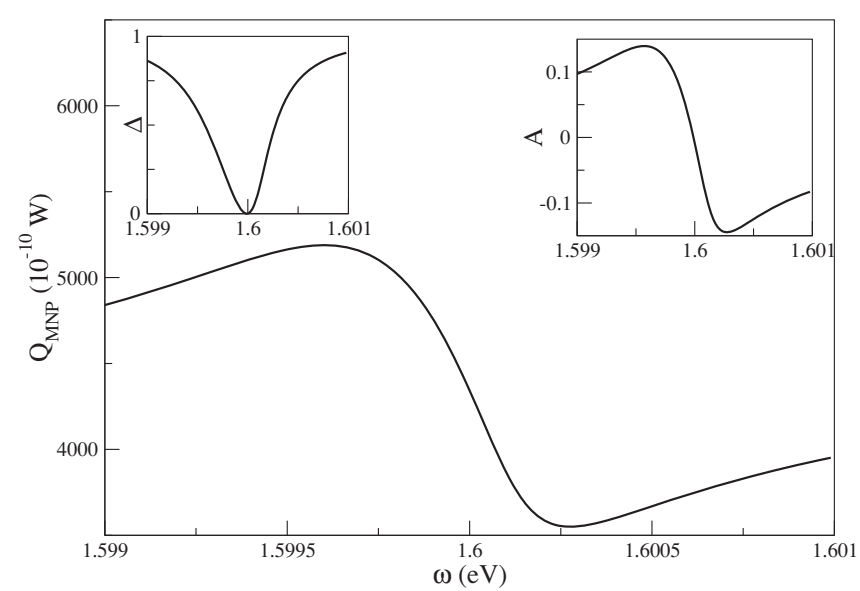

FIG. 8. Bright state, with an exciton energy level at $1.6 \mathrm{eV}, L=$ $70 \mathrm{~nm}, w=14 \mathrm{~nm}, \mu=0.5 e \mathrm{~nm}$. Absorption rate of the MNP, $Q_{\mathrm{MNP}}$, population difference $\Delta$, and the real part of the SQD dipole moment $A$ all show a very strong and broad response of the system. 


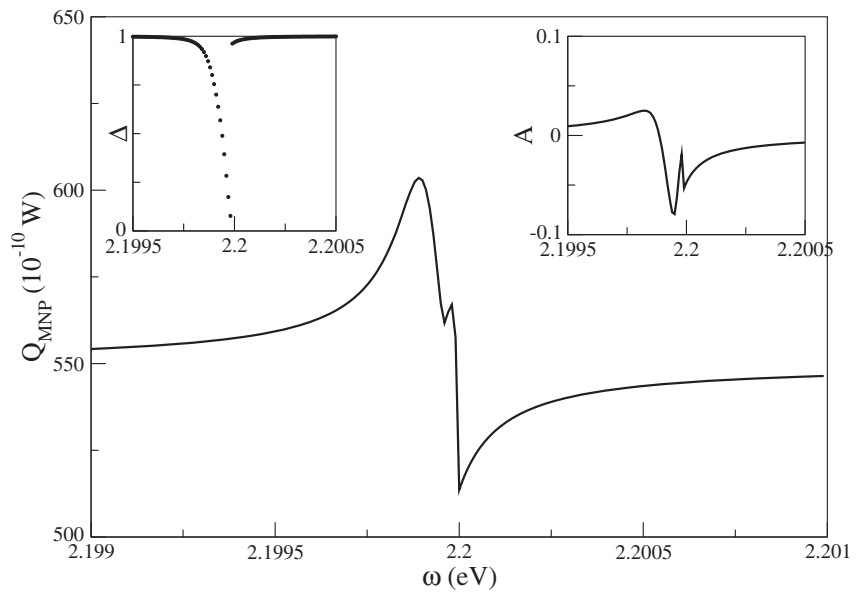

FIG. 9. Dark state, with an exciton energy level at $2.2 \mathrm{eV}, L=$ $70 \mathrm{~nm}, w=14 \mathrm{~nm}, \mu=0.5 e \mathrm{~nm}$. Absorption rate of the MNP, $Q_{\mathrm{MNP}}$, and the real part of the SQD dipole moment $A$ both show a bistability in the system. The population difference $\Delta$ shows a discontinuity and strong suppression in the excitation of the SQD.

\section{Dark state}

As shown in Fig. 6, the dark state is characterized by a large response in the MNP due to the dipole of the SQD, but with little response to the plane wave. When we choose our exciton resonance to coincide with the frequency of the dark state, we see that the SQD responds strongly to the self-interaction. Because the suppression regime as well as the bistable regime are controlled by the self-interaction strength relative to the coupling to the applied plane wave, both regimes of behavior are very easy to reach in this case.

With a SQD dipole moment of only $\mu=0.5 e \mathrm{~nm}$, we see very strong suppression in the SQD response as well as the beginnings of bistability (see Fig. 9), whereas with a spherical MNP with radius $7 \mathrm{~nm}$ we would need a SQD with a dipole moment of $\mu=3 e \mathrm{~nm}$ to elicit a similar response. ${ }^{10}$

Comparing the response of the dark state to that of the bright state, we see that the MNP absorption is an order of magnitude greater for the bright state. For both the bright and dark states we have a Fano resonance in $Q_{\mathrm{MNP}}$, which, according to our previous investigations, indicated the weakest level of coupling between the MNP and SQD. However, suppression and bistability of the SQD response evident in Fig. 9 would indicate the strongest-coupling regime. Thus, we see that these different types of hybrid behavior can coexist.

\section{B. Exciton-induced transparency}

For a spherical MNP in the dipole limit, the appearance of an exciton-induced transparency (EXIT) is determined by the relation

$$
\mu(a) \geqslant-\frac{2 \pi \epsilon_{B} \epsilon_{\mathrm{effS}} R^{3} E_{0}}{s_{\alpha} A},
$$

as shown in Ref. 9, which defines when the field from the SQD cancels the applied field inside the MNP. Note that this approximation assumes that the field inside the MNP is constant and equal to the value at the center of the MNP. It is then worth showing that this feature does in fact survive when
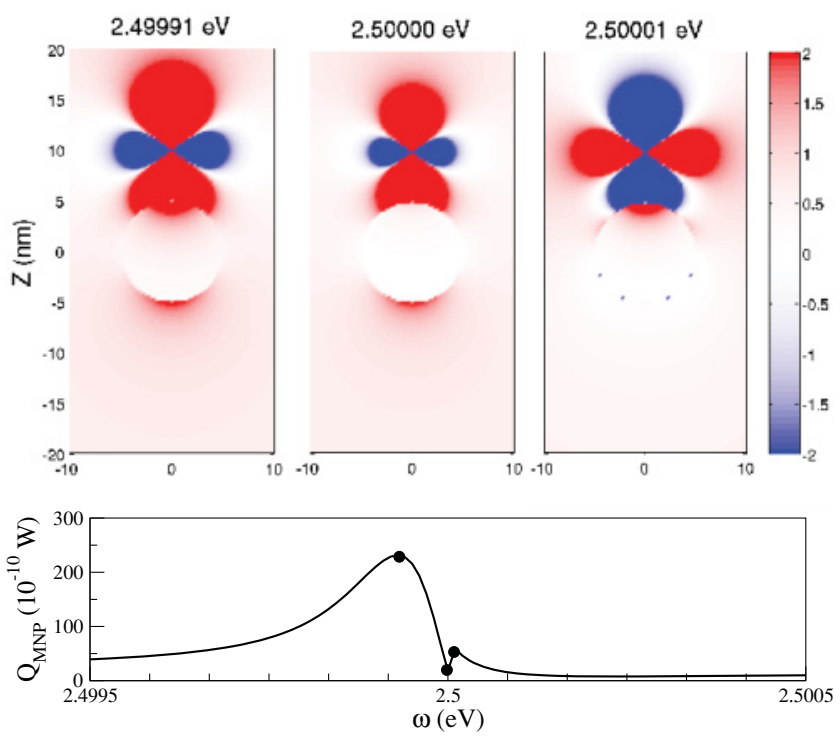

FIG. 10. (Color online) (Top) Near field of a 5-nm radius spherical MNP interacting with a SQD located $10 \mathrm{~nm}$ away from center for three values of applied frequency. Shown in color is the $z$ component of the electric field. The first plot shows a strong dipole field from the SQD, in phase with the applied field that penetrates the MNP to a depth of $\approx 2 \mathrm{~nm}$. The middle plot show the system at resonance and the appearance of the exciton-induced transparency in the MNP. The third plot shows a strong dipole field from the SQD, now out of phase with the applied field, that again penetrates the MNP to a depth of $\approx 1 \mathrm{~nm}$. (Bottom) $Q_{\mathrm{MNP}}$ is shown for the three frequencies featured above.

a more thorough calculation is performed for a MNP sphere utilizing a full electrodynamical calculation using the BEM.

For a spherical MNP with $a=7 \mathrm{~nm}$ coupled to a SQD with a dipole moment of $\mu=1 e \mathrm{~nm}$, we plot the $z$ component of the electric field (see Fig. 10). When the system is below resonance, we see a large response from the SQD as its dipole moment is very large and in phase with the applied field. We also see the dipole field extend into the MNP to a depth of $\approx 2 \mathrm{~nm}$. At resonance, as the off-diagonal density-matrix elements are nearly zero, the SQD dipole moment is much smaller, and we see a weaker dipole moment in the SQD. Also, the interference inside the MNP now causes a near cancellation of the field inside the MNP and we see an excitoninduced transparency as we have previously predicted. ${ }^{9}$ Above resonance, the dipole field of the SQD is once again strong, but is now out of phase with the applied field.

For nanorods, the appearance of EXIT is problematic. From the relation describing the appearance of EXIT [Eq. (9)], we expect that, with the large center-to-center separation inherent when using a nanorod as the MNP, EXIT will be attainable only for extremely large values of the SQD dipole moment. Also, the spatial variation of the field over a nanorod MNP is not properly accounted for in the dipole limit (which was used to generate this relation). When we performed the full calculations, we find that EXIT is very difficult to produce in a system with a nanorod. The spatial variation of the dipole field (from the SQD), over the length of a nanorod, can no longer cancel out the constant applied field inside the MNP. The absorption still has a Fano-like line shape, but now the 
magnitude of the interference is too small in comparison to the applied field in order to produce a transparency.

\section{CONCLUDING REMARKS}

We have studied hybrid molecule structures made from a metal nanoparticle nanorod and a quantum dot to show how MNP geometry can be used to tailor local fields, coupling, and dynamics of these hybrid structures. We have employed a full electrodynamical calculations of the near fields for MNP-SQD hybrid molecules based on the boundary element method. The method allows for tackling more complex geometries with a full description of the retarded multipolar optical response. Calculations performed on systems consisting of a SQD and a nanorod MNP have shown that a broad range of system behavior can be reached experimentally much more easily than previously thought. In particular, we find that the response of the system could be tailored by engineering a metal nanoparticle shape and the placement of SQDs on the MNP to control the individual local near fields that couple the MNPs and SQDs. We identify regimes where dark modes can influence hybrid response. Strong local-field coupling via dark modes changes the interference and self-interaction effects dramatically. External fields do not directly drive this MNP dark mode, so SQD-MNP coupling is dominated by the local induced coupling. All of these aspects could enhance the capabilities of metal nanostructures to provide spatial and spectral control of the optical properties of single emitters. *artuso@umd.edu

${ }^{1}$ T. Pons, I. L. Medintz, K. E. Sapsford, S. Higashiya, A. F. Grimes, D. S. English, and H. Mattoussi, Nano Lett. 7, 3157 (2007).

${ }^{2}$ A. V. Akimov, A. Mukherjee, C. L. Yu, D. E. Chang, A. S. Zibrov, P. R. Hemmer, H. Park, and M. D. Lukin, Nature (London) 450, 402 (2007).

${ }^{3}$ Y. Fedutik, V. V. Temnov, O. Schops, U. Woggon, and M. V. Artemyev, Phys. Rev. Lett. 99, 136802 (2007).

${ }^{4}$ H. Mertens, J. S. Biteen, H. A. Atwater, and A. Polman, Nano Lett. 6, 2622 (2006).

${ }^{5}$ Alberto G. Curto, Giorgio Volpe, Tim H. Taminiau, Mark P. Kreuzer, Romain Quidant, and Niek F. van Hulst, Science 329, 930 (2010).

${ }^{6}$ E. Altewischer, M. P. van Exter, and J. P. Woerdman, Nature (London) 418, 304 (2002).

${ }^{7}$ Sylvain Fasel, Franck Robin, Esteban Moreno, Daniel Erni, Nicolas Gisin, and Hugo Zbinden, Phys. Rev. Lett. 94, 110501 (2005).

${ }^{8}$ Wei Zhang, Alexander O. Govorov, and Garnett W. Bryant, Phys. Rev. Lett. 97, 146804 (2006).

${ }^{9}$ Ryan D. Artuso and Garnett W. Bryant, Nano Lett. 8, 2106 (2008).

${ }^{10}$ Ryan D. Artuso and Garnett W. Bryant, Phys. Rev. B 82, 195419 (2010).

${ }^{11}$ Jie-Yun Yan, Wei Zhang, Suqing Duan, Xian-Geng Zhao, and Alexander O. Govorov, Phys. Rev. B 77, 165301 (2008).

${ }^{12}$ S. M. Sadeghi, Nanotechnology 21, 355501 (2010).
${ }^{13}$ A. Govorov, G. Bryant, W. Zhang, T. Skeini, J. Lee, N. Kotov, J. Slocik, and R. Naik, Nano Lett. 6, 984 (2006).

${ }^{14}$ J. Lindberg, K. Lindfors, T. Setälä, and M. Kaivola, J. Opt. Soc. Am. A 24, 3427 (2007).

${ }^{15}$ S. M. Sadeghi, L. Deng, X. Li, and W.-P. Huang, Nanotechnology 20, 365401 (2009).

${ }^{16} \mathrm{Mu}-$ Tian Cheng, Shao-Ding Liu, Hui-Jun Zhou, Zhong-Hua Hao, and Qu-Quan Wang, Opt. Lett. 32, 2125 (2007).

${ }^{17}$ David Dzsotjan, Anders S. Sørensen, and Michael Fleischhauer, Phys. Rev. B 82, 075427 (2010).

${ }^{18}$ A. Gonzalez-Tudela, D. Martín-Cano, E. Moreno, L. MartínMoreno, C. Tejedor, and F. J. García-Vidal, Phys. Rev. Lett. 106, 020501 (2011)

${ }^{19}$ R. Esteban, T. V. Teperik, and J. J. Greffet, Phys. Rev. Lett. 104, 026802 (2010).

${ }^{20}$ Diego Martíln-Cano, Luis Martín-Moreno, Francisco J. GarcíaVidal, and Esteban Moreno, Nano Lett. 10, 3129 (2010).

${ }^{21}$ M. Liu, T. W. Lee, S. K. Gray, P. Guyot-Sionnest, and M. Pelton, Phys. Rev. Lett. 102, 107401 (2009).

${ }^{22}$ A. Yariv, Quantum Electronics (Wiley, New York, 1975).

${ }^{23}$ P. B. Johnson and R. W. Christy, Phys. Rev. B 6, 4370 (1972).

${ }^{24}$ K. L. Silverman, R. P. Mirin, S. T. Cundiff, and A. G. Norman, Appl. Phys. Lett. 82, 4552 (2003).

${ }^{25}$ T. H. Stievater, Xiaoqin Li, D. G. Steel, D. Gammon, D. S. Katzer, D. Park, C. Piermarocchi, and L. J. Sham, Phys. Rev. Lett. 87, 133603 (2001). 